\title{
INFLUENCIA DEL USO DE ESTRATEGIAS DE APRENDIZAJE Y MOTIVACIÓN EN EL NIVEL DE HABILIDADES INVESTIGATIVAS EN ESTUDIANTES DE POSGRADO EN ODONTOLOGÍA
}

\author{
INFLUENCE OF THE USE OF LEARNING STRATEGIES IN THE LEVEL OF RESEARCH \\ SKILLS IN POSTGRADUATE STUDENTS IN DENTISTRY
}

\author{
Dahiana Alfaro-Carballido ${ }^{1 a b d}$, Alberto Quitzgaard-Álvarez ${ }^{1 e d}$, Janet Guevara-Canales $^{1 a b d}$, Rafael Morales-Vadillo ${ }^{1 a c d}$, \\ Hans Morgenstern-Orezzolli ${ }^{1 a b f}$
}

\begin{abstract}
RESUMEN
Objetivo: Determinar si el uso de estrategias de aprendizaje y motivación influyen en el nivel de habilidades investigativas en estudiantes de posgrado en odontología de la Universidad de San Martín de Porres. Materiales y métodos: se encuestaron 130 estudiantes con una tasa de no respuesta del $16.07 \%$, los cuales pertenecen a los programas de maestría y especialidades, seleccionados en forma aleatoria. Se utilizó dos cuestionarios: 1) de estrategias de aprendizaje y motivación; y 2) de habilidades investigativas. Resultados: se realizó el análisis descriptivo de los datos, Chi ${ }^{2}$ y ANOVA para evaluar la asociación entre las variables y la razón de prevalencia con la prueba de regresión lineal de Poisson. Se encontró asociación en cuanto a las variables tiempo de estudio, trabajos científicos previos, pertenencia a una asociación científica, cursos anteriores de investigación y métodos de estudio Chi2 $(p<0.05)$, también se encontró asociación con la motivación y estrategias de aprendizaje ANOVA $(p<0.05)$ resaltando la motivación interna del estudiante y el pensamiento crítico en todas las etapas de investigación $(p<0.05)$. Conclusiones: se encontró que los estudiantes que tienen un nivel alto de uso de estrategias de aprendizaje y motivación tienen casi tres veces más probabilidades de tener habilidades investigativas altas (RP:2.82 IC=1.55-5.14), cuentan con un nivel de habilidades investigativas entre medio a alto (58\%).
\end{abstract}

Palabras Clave: Motivación, aprendizaje, técnicas de investigación, investigación. (Fuente: DeCS BIREME)

\begin{abstract}
Objetive: To determine if the use of learning strategies and motivation influence the level of research skills in postgraduate students in dentistry of the Universidad de San Martín de Porres. Materials and Methods: 130 students were surveyed with a non-response rate of $16.07 \%$ which belong to the programs of masters and specialities, which were randomly selected. We used two questionnaires 1) learning strategies and motivation and 2) questionnaire to evaluate the rsearch skills which was previously validated. Results: Descriptive analysis of the data, Chi2 and ANOVA was carried out to evaluate the association between the variables and the ratio of prevalence with the linear regression test of Poison. An association was found regarding the variables study time, previous scientific work, membership of a scientific association and antecedets of research and methods of study courses Chi2 $(p<0.05)$, we also found association with motivation and learning strategies ANOVA $(p<0.05)$ highlighting Internal motivation of the student and critical thinking in all stages of research $(p<0.05)$. Conclusions: It was found that students who have a high level of use of learning strategies and motivation are almost three times more likely to have high research skills (RP: $2.82 \mathrm{Cl}=1.55-5.14$ ), Have a medium to high level of research skills $(58 \%)$
\end{abstract}

Keywords: Motivation, learning, investigation, research dental. (Source: MeSH NLM)

Recibido: 18 de setiembre de 2017

Aprobado: 13 de diciembre de 2017

Publicado: 30 de marzo de 2018

${ }^{1}$ Universidad de San Martin de Porres

${ }^{\text {a }}$ Cirujano Dentista; b Doctor en Odontologia

c Doctor en Educacion; ${ }^{\mathrm{d}}$ Docente de Posgrado

e Psicopedagogo, Doctor en Psicologia

f Maestro en Educacion

Correspondencia:

Dahiana Alfaro Carballido

Dirección: Calle Crepúsculo 104, dpto. 301.Lima. Teléfono: 987768124

Correo electrónico: :lalfaroc@usmp.pe
Este es un artículo Open Access distribuido bajo la licencia Creative Commons Atribución-NoComercial- Compartir Igual 4.0

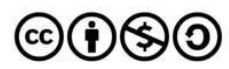

Citar como: Alfaro- Carballido D, Quitzgaard-Álvarez A, Guevara Canales J, Morales Vadillo R, Morgenstern Orezolli H. Odontología basada en evidencia: conceptos generales y su relevancia. KIRU. 2018; 15(1): 8-19. https://doi.org/10.24265/kiru.2018.v15n1.01 


\section{INTRODUCCIÓN}

Los avances que se están dando en la educación superior, abarcan distintos campos de las ciencias de la salud, dentro de ellas la odontología, que cada día busca adaptar las enseñanzas universitarias a estándares internacionales, mostrando un cambioen la concepción y modo de abordar el aprendizaje, donde prima la innovación y una mejor gestión del conocimiento, teniendo a la investigación científica como base para la formación de profesionales de calidad (1).

La American Dental Association (ADA), ha revisado los criterios estándares de acreditación internacional para los programas de educación dental donde incluye la integración de la investigación, su evaluación y garantía de que los estudiantes reciban información y guía de las oportunidades en realizar investigación dentro de sus programas de entrenamiento, (2-3) así la Facultad de Odontología de la Universidad de San Martín de Porres (FO-USMP) dentro del marco de desarrollo de los programas de posgrado que ofrece en la carrera de Odontología, tiene como objetivo fundamental formar maestros y especialistas en odontología de acuerdo a estándares nacionales e internacionales, altamente competitivos, con capacidad de liderazgo y comprometidos con la realidad nacional del país, la cual viene siendo respaldada con dos acreditaciones internacionales con la Agencia para la Calidad del Sistema Universitario de Galicia ACSUG (España), Instituto Latinoamericano de Acreditación en Odontología ILAO (Brasil) y el Sistema Nacional de Evaluación, Acreditación y Certificación de la Calidad Educativa (SINEACE) y su licenciamiento con Superintendencia Nacional de Educación Superior Universitaria SUNEDU (Perú), cumpliendo con la principal función de la Universidad que es hacer investigación y vincular a la sociedad con la generación de nuevos conocimientos ${ }^{(4-8)}$.

Actualmente las recientes investigaciones en educación en odontología resaltan que dentro del perfil profesional del cirujano dentista, se debe contar con habilidades investigativas que le permitan desarrollar el juicio crítico, reflexivo y creativo para poder resolver problemas de la salud bucal y salud general que se presenten en su práctica clínica diaria. Este proceso de formación y desarrollo de habilidades investigativas en los estudiantes involucra a todas las disciplinas y asignaturas que conforman el currículo en posgrado en odontología con una visión interdisciplinaria, multidisciplinaria y transdisciplinaria ${ }^{(3,8,9)}$.

Investigar es un proceso complejo, que implica necesariamente una secuencia operacional sistemática que motive al estudiante al desarrollo de habilidades para desarrollarla, es allí, donde el uso de estrategias de aprendizaje y motivación es de suma importancia en el desarrollo de estas competencias y fundamental en el rol docente para poder orientar al estudiante al aprendizaje autónomo y significativo(107).

Hoy en día la motivación y el uso de estrategias de aprendizaje son áreas bastante estudiadas en el campo de la educación y psicología educativa, ya que se ha sugerido que tiene un papel fundamental para el éxito académico de los estudiantes, su bienestar y para los resultados clínicos en los pacientes. ${ }^{1,9}$ Existen muchos estudios que demuestran cómo estas variables influyen en el rendimiento académico y performance de los estudiantes, ${ }^{(9-19)}$ pero ninguno que se haya realizado a nivel de las habilidades investigativas, por lo que el propósito del estudio fue determinar si el uso de estrategias de aprendizaje y motivación influyen en el nivel de habilidades investigativas en estudiantes de posgrado en odontología de la USMP y sus factores asociados.

\section{MATERIALES Y MÉTODO}

El presente estudio se ubica en el área de ciencias básicas, descriptivo analítico y transversal. La población de estudio estuvo constituida por todos los estudiantes de posgrado de la FO-USMP matriculados en los programas de especialidad y maestrías el semestre 2016-II, se encuestaron a 130 estudiantes, procesando 112 cuestionarios, teniendo una tasa de no respuesta del $16.07 \%$. Se contó con la aprobación del proyecto por la unidad de posgrado de la FO-USMP y se cumplieron con las normas bioéticas que implican a la investigación científica.

\section{Validez y confiablidad de los instrumentos:}

Se utilizaron dos cuestionarios: 1) el cuestionario de estrategias de aprendizaje y motivación (CEAM) y 2) el Cuestionario de Habilidades Investigativas ( $\mathrm{CHI}$ ) validados en un estudio piloto previo realizado en la Universidad Nacional Mayor de San Marcos (UNMSM) a 52 estudiantes, que permitió valorar la viabilidad, validez y confiabilidad de los instrumentos ${ }^{(20)}$.

El CEAM consta de 82 ítems y dos escalas una en referencia a la motivación que se subdivide en cinco dimensiones: motivación extrínseca, motivación intrínseca, valor de la tarea, confianza, eficacia personal y otra escala que evalúa las estrategias de aprendizaje que se subdivide en nuevedimensiones: el ensayo, la elaboración, la organización, pensamiento crítico, autorregulación metacognitiva, 
manejo de recursos, regulación al esfuerzo aprendizaje en paralelo y búsqueda de ayuda. Las alternativas de respuesta fueron de tipo Likert en una escala de cinco puntos (1 nunca, 2 pocas veces, 3 algunas veces, 4 muchas veces, 5 siempre). Se aplicó la versión en español del cuestionario ya utilizada y validada a nuestro contexto por Villegas y cols. ${ }^{(12)}$ obteniendo un valor de Alfa de Cronbach en el piloto de 0,885 para todo el cuestionario.

El CHI está compuesto de 45 ítems subdividido en tres dimensiones según las etapas de investigación: planificación, ejecución y difusión de acuerdo al nivel alcanzado bajo, medio y alto; para la elaboración de los ítems del cuestionario de habilidades investigativas se realizó una evaluación y análisis crítico de la literatura científica actualizada sobre el tema, la validez de contenido se realizó mediante un juicio de 11 expertos con el grado de doctor y especialistas con experiencia en investigación científica y educación obteniendo un valor de $\mathrm{V}$ de Aiken de 0,98. El análisis de constructo se realizó aplicando el análisis factorial con la prueba de KaiserMeyer-Olkin $=0,534 \mathrm{y}$ prueba de Esfericidad de Bartlett resultando $p<0,001$ teniendo como resultado concordancia entre componentes, un Alfa de Cronbach de 0,967 para todo el cuestionario y 0,914 ; 0,948 y 0,909 respectivamente para cada dimensión, correlación de Spearman-Brown entre ítems de 1 $(p<0,001)$ lo que indica una alta confiabilidad $y$ consistencia interna entre ítems. ${ }^{21}$

\section{Aplicación final de los instrumentos}

Ambos cuestionarios fueron aplicados a los estudiantes del posgrado en Odontología de la USMP en el periodo de agosto a noviembre del2016 en sus aulas de clase en un tiempo aproximado de 25 a 30 minutos, previo consentimiento informado.

\section{Procesamiento de la información}

Se realizó con el programa estadístico SPSS versión 23, el análisis descriptivo mediante la obtención de

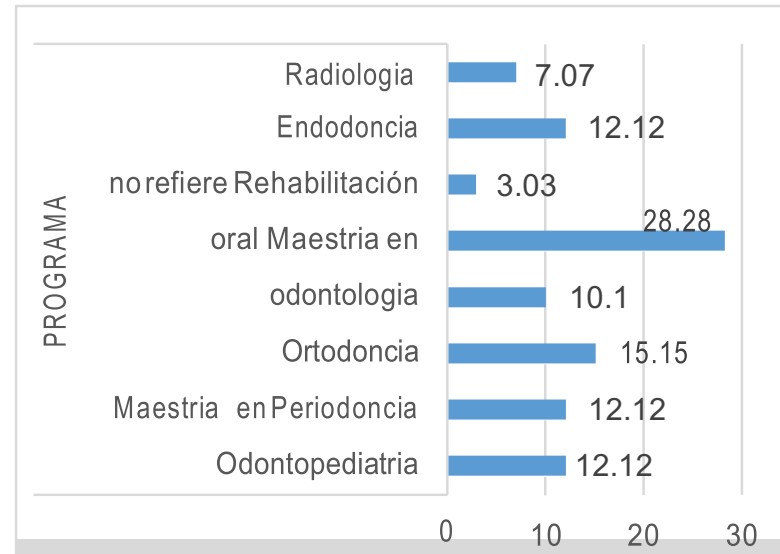

Figura 1. Programas de posgrado en las frecuencias absolutas y relativas de las variables cualitativas y el promedio y desviación estándar de las variables cuantitativas. El análisis bivariado se desarrolló mediante las pruebas de Chi cuadrado y ANOVA, demostrándose la normalidad de los datos cuantitativos mediante la prueba de Kolmogorov Smirnof. La obtención de la razón de prevalencia se realizó con la prueba de regresión lineal de Poisson. El estudio contó con un nivel de confianza del 95\% y un $\mathrm{p}<0.05$.

\section{RESULTADOS}

La mayoría de estudiantes entrevistados son del género femenino en un $58.04 \%$ y solteros (92\%), con una edad promedio de 28 años, pertenecientes al programa de posgrado en odontologia (Figura 1) programa de especialidad (88.88\%) y el $22.12 \%$ a maestrías, distribuidos equitativamente por ciclo académico( I ciclo $29 \%$, II ciclo $20 \%$, III ciclo $28 \%$, IV ciclo $21 \%$ y no refiere $2 \%$ ), estudiantes de posgrado procedentes en su mayoría de la Universidad de San Martin de Porres (86.61\%). En cuanto a las características educacionales la mayoría de estudiantes refirió trabajos de investigación previos siendo más frecuentes los posters y mesas clínicas con un $45.62 \%$. Solo 22 estudiantes (19.30\%) son asociados a una sociedad científica y el $28.07 \%$ refieren haber llevado adicionalmente cursos de metodología y métodos de estudio, el $56.14 \%$ de estudiantes maneja el idioma inglés como segunda lengua seguido del portugués y francés y el interés por realizar investigaciones es bastante alto (73.68\%). (Figuras 1 - 8).

El nivel de habilidades investigativas en estudiantes del posgrado en odontología esta entre medio y alto con un valor de $57.88 \%$. Según etapas se encontró un mejor nivel de habilidades investigativas en la etapa de planificación $(68.42 \%)$ seguida de la etapa de ejecución con un $65.79 \%$ y la más baja la etapa de difusión (59.39\%). (Figura 9 y 10)

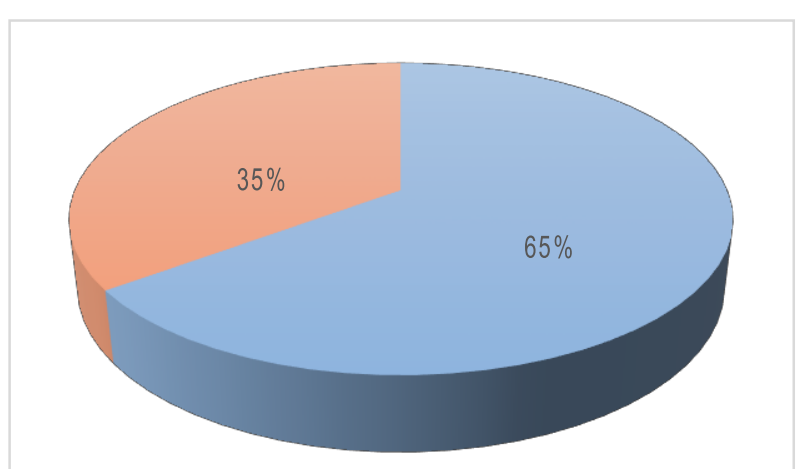

Trabajoscientíficossi Trabajos científicosno Figura 2. Porcentaje de participación en la presentación de trabajos científicos 

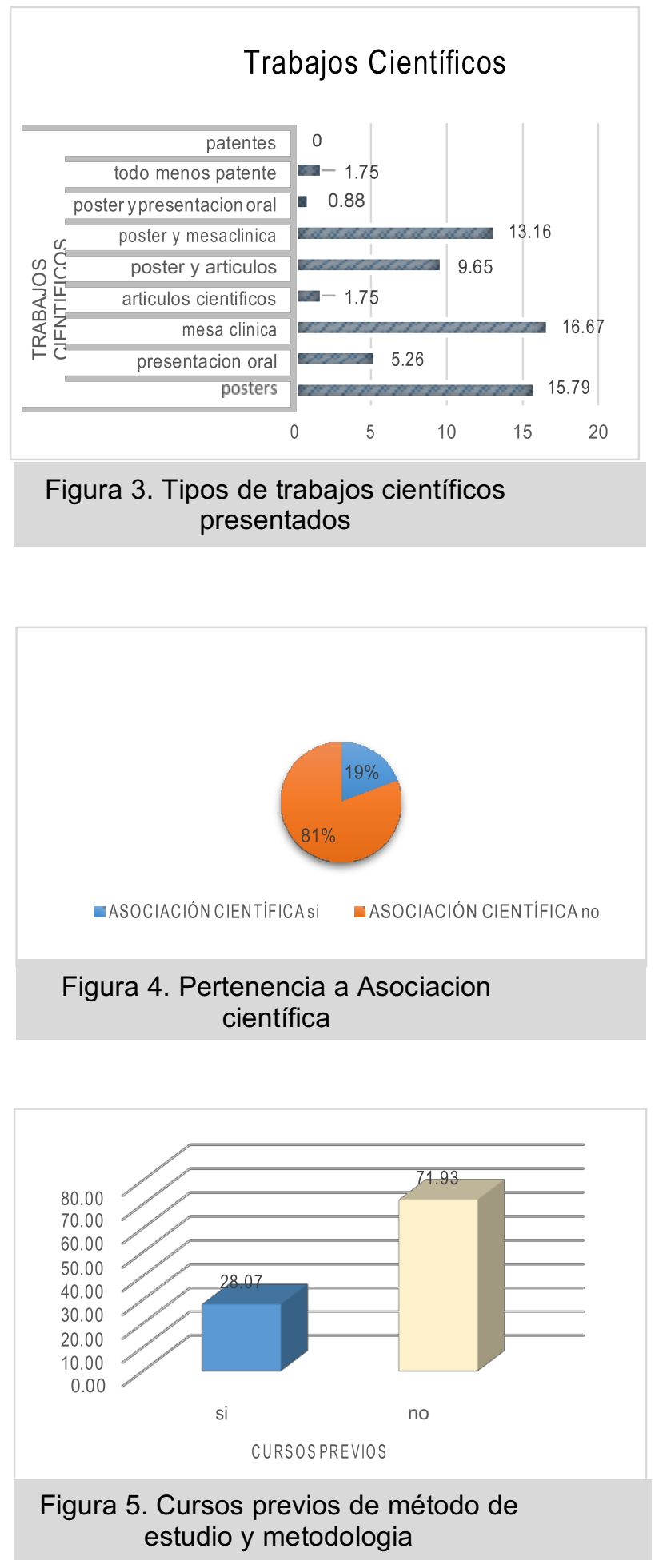

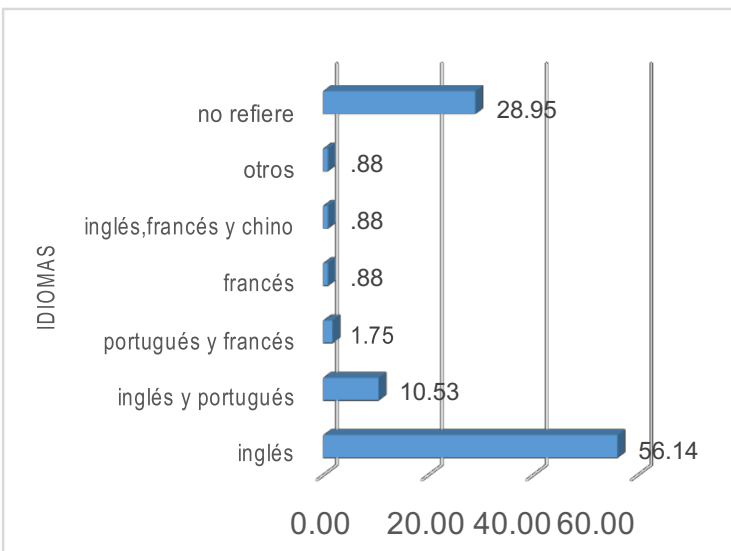

Figura 6. Porcentaje de estudiantes que hablan idiomas

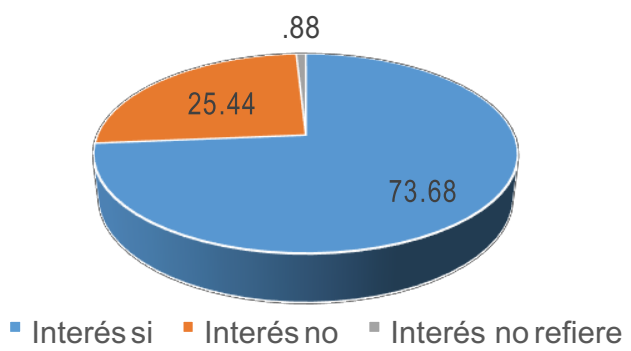

Figura 7. Interes por realizar una investigación cientifica

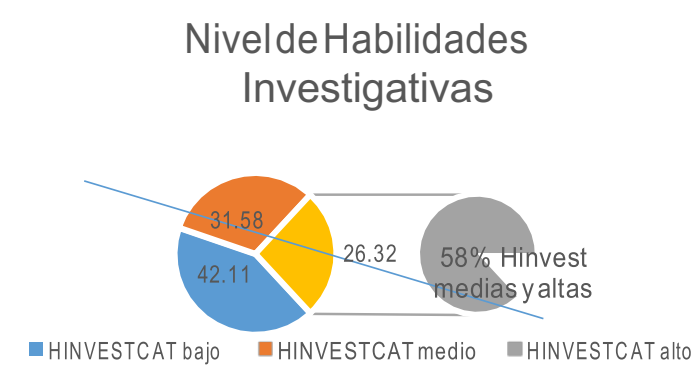

Figura 8. INivel de habilidades investigativas 


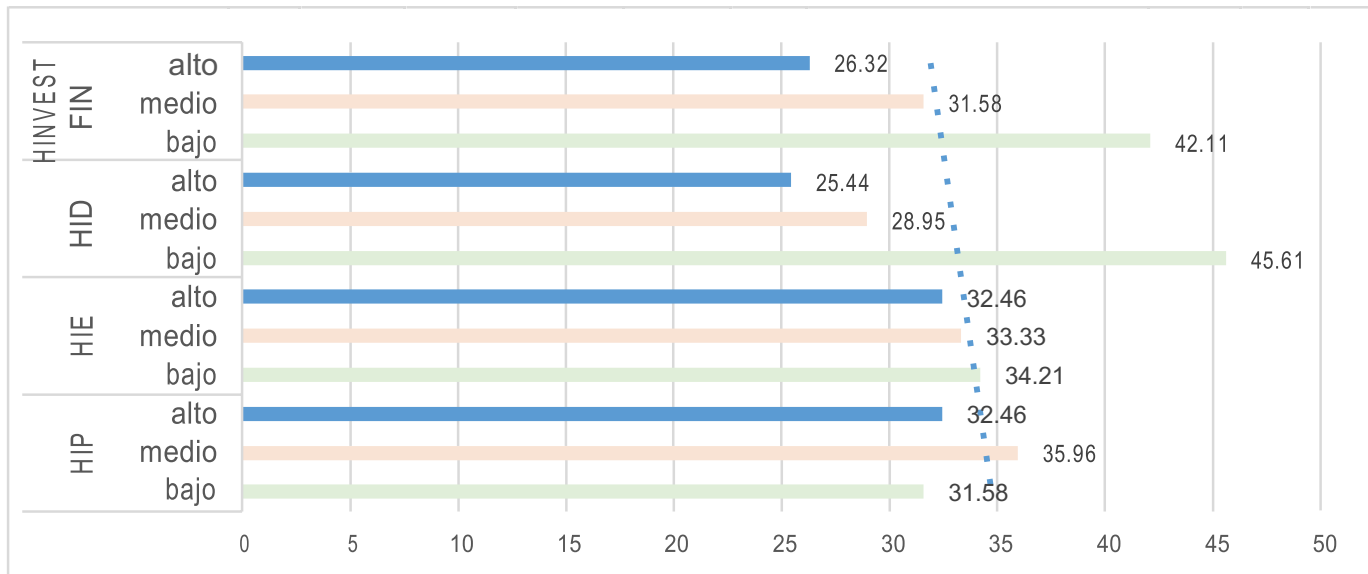

Figura 9. Nivel de habilidades investigativas según etapas de investigación

Los ítems mejor valorados fueron el $4,7,11$ y 28 $(83.03 \%, 86.00 \%, 91.07 \%, 90.18 \%)$ correspondiente a la formulación del problema de investigación, objetivos, uso de bases de datos especializadas y manejo de programas de computador. (Tabla 1) .

El cuestionario CEAM completo se valoró como alto con una media de 535. 98, la dimensión motivación como alta (x:197.18) seguido de la dimensión estrategias de aprendizaje como alta (x:170.89). Dentro de la dimensión motivación, las subescalas mejor valoradas fueron eficacia personal (x: 59.65) y el valor de la tarea (x: 40.93), la motivación interna fue mejor valorada ( $x: 36.71)$ a diferencia de la motivación externa con un promedio de 30.28. En la dimensión estrategias de aprendizaje las mejor valoradas fueron las estrategias metacognitivas ( $x: 218.38)$ a diferencia de las estrategias de gestión de recursos con un promedio de 120.42. Dentro de las estrategias metacognitivas sobresalen las subescalas de metacognitivas (x:83.91), elaboración (x:44.33) y pensamiento crítico (34.31) y dentro de la escala de gestión de recursos las subescalas mejor valoradas fueron las de tiempo y ambiente (x:53.77) y regulación al esfuerzo (x:31.53), siendo el aprendizaje en paralelo mejor valorado $(x: 20.16)$ que la búsqueda de ayuda con un promedio de (x:14.96). (IFigura 10)

Asimismo, se encontró asociación en el nivel de habilidades investigativas de planificación y ejecución con la motivación y con las subescalas: motivación interna $(p<0.001)$, motivación externa $(p=0.01)$, valor de la tarea $(p=0.01)$, confianza del aprendizaje $(p<0,001)$ y eficacia del aprendizaje $(p<0,001)$ ANOVA $p<0.05)$. (Tabla 2 )

Tabla 1.Habilidades investigativas mejor valoradas por los estudiantes de posgrado en odontología de la USMP según etapas.

\begin{tabular}{lcccl}
\hline Dimensiones & Ítems & $\mathrm{n}$ & $\%$ & \\
\hline Etapa de planificación & 11 & 102 & 91.07 & Utiliza bases de datos especializadas \\
& 7 & 97 & 86.62 & Formula objetivos de estudio de una investigación \\
& 4 & 93 & 83.03 & Desarrolla el planteamiento del problema de investigación \\
Etapa de ejecución & 10 & 88 & 78.87 & Utiliza organizadores, esquemas, imágenes para sintetizar información \\
& 28 & 101 & 90.18 & Maneja programas de computador \\
Etapa de difusión & 34 & 92 & 82.24 & Precisa adecuadamente sus conclusiones \\
& 33 & 90 & 80.72 & Discute coherentemente sus resultados \\
& 35 & 89 & 79.36 & Plantea soluciones creativas y lógicas a la problemática de estudio \\
& 44 & 86 & 77.47 & Domina técnicas de expresión oral para sustentar su trabajo \\
& 43 & 84 & 75.76 & Articula conocimientos con facilidad para elaborar un poster \\
& 42 & 83 & 74.78 & Usa adecuadamente la tecnología para elaborar presentaciones de impacto \\
\hline
\end{tabular}



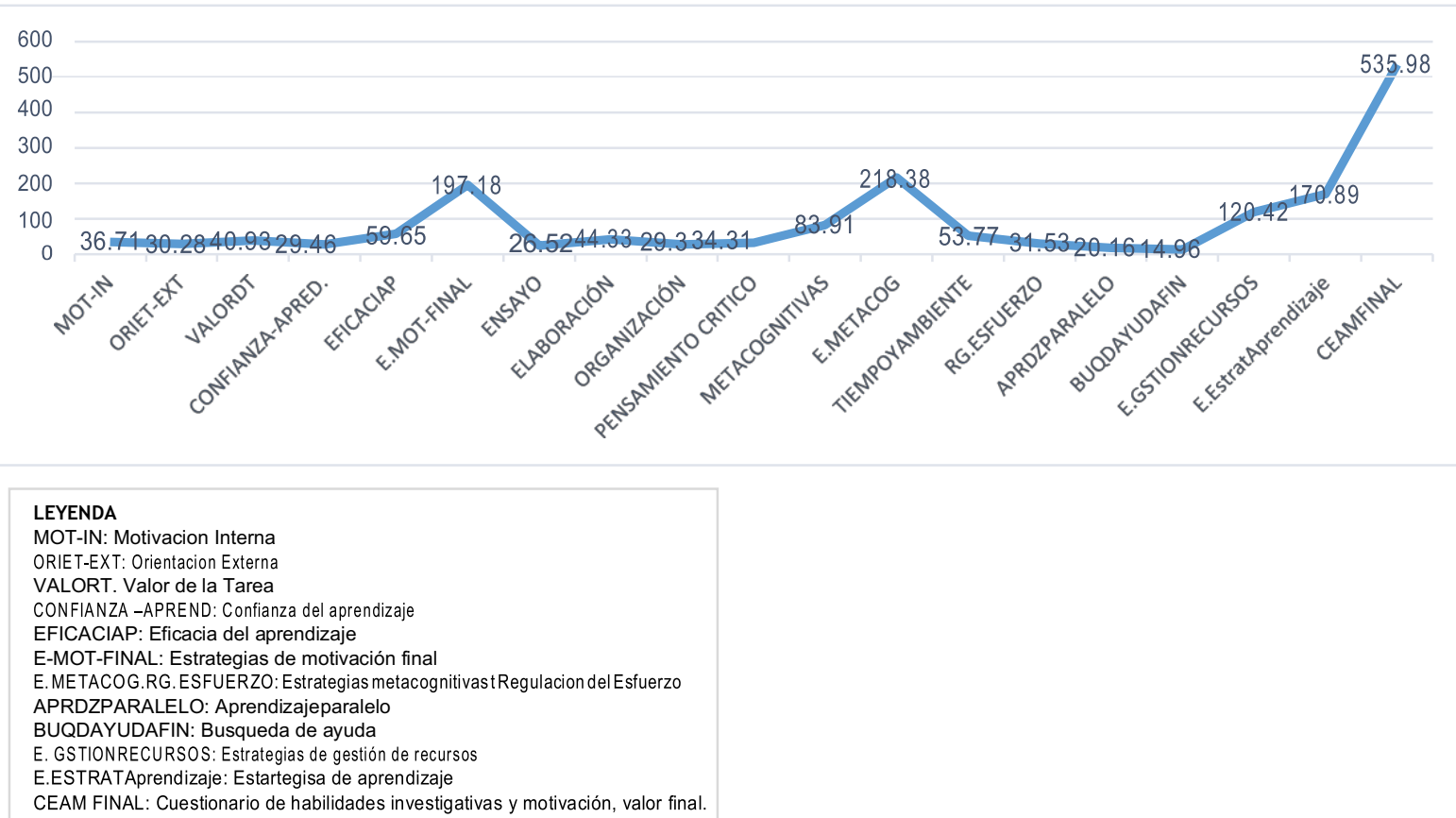

Figura 10. Estrategias de aprendizaje

Tabla 2. Asociación del uso de habilidades investigativas y motivación de los estudiantes de posgrado en odontología de la FO-USMP

\begin{tabular}{|c|c|c|c|c|c|c|c|c|c|c|c|c|c|c|c|c|}
\hline & & MOT-IN & & & $\begin{array}{c}\text { ORIET- } \\
\text { EXT }\end{array}$ & & & $\begin{array}{l}\text { VALO } \\
\text { RDT }\end{array}$ & & & $\begin{array}{c}\text { CONFIANZA } \\
\text {-APRED. }\end{array}$ & & & $\begin{array}{l}\text { EFICAC } \\
\text { IAP }\end{array}$ & & \\
\hline & & Media & $\begin{array}{c}\text { Desviación } \\
\text { estándar }\end{array}$ & $p$ & Media & $\begin{array}{c}\text { Desviación } \\
\text { estándar }\end{array}$ & $p$ & Media & $\begin{array}{c}\text { Desviación } \\
\text { estándar }\end{array}$ & $p$ & Media & $\begin{array}{c}\text { Desviación } \\
\text { estándar }\end{array}$ & $\mathrm{p}$ & Media & $\begin{array}{c}\text { Desviación } \\
\text { estándar }\end{array}$ & $p$ \\
\hline \multirow[t]{3}{*}{ HIPCAT } & bajo & 16.72 & 3.24 & & 13.97 & 3.78 & & 19.06 & 5.35 & & 14.14 & 2.86 & & 28.44 & 5.54 & \\
\hline & medio & 18.56 & 2.58 & 0.00 & 15.56 & 2.97 & 0.01 & 20.66 & 2.37 & 0.01 & 14.39 & 3.14 & 0.00 & 29.07 & 6.2 & 0.00 \\
\hline & alto & 20.22 & 2.42 & & 16.22 & 2.68 & & 21.95 & 3 & & 16.08 & 2.09 & & 32.81 & 4.11 & \\
\hline \multirow[t]{3}{*}{ HIECAT } & bajo & 16.79 & 3.12 & & 14.03 & 3.67 & & 19.18 & 5.18 & & 14.18 & 2.84 & & 28.36 & 5.37 & \\
\hline & medio & 18.63 & 2.67 & 0.00 & 15.63 & 3.03 & 0.01 & 20.66 & 2.41 & 0.01 & 14.37 & 3.18 & 0.01 & 29.21 & 6.39 & 0.00 \\
\hline & alto & 20.22 & 2.42 & & 16.22 & 2.68 & & 21.95 & 3 & & 16.08 & 2.09 & & 32.81 & 4.11 & \\
\hline \multirow[t]{3}{*}{ HIDCAT } & bajo & 18.63 & 2.85 & & 15.42 & 2.95 & & 20.96 & 2.91 & & 14.6 & 3.15 & & 29.37 & 6.32 & \\
\hline & medio & 18.03 & 4.03 & 0.53 & 15.09 & 3.75 & 0.9 & 19.3 & 5.51 & 0.08 & 15.03 & 3.16 & 0.66 & 29.97 & 6.17 & 0.26 \\
\hline & alto & 18.86 & 2.07 & & 15.21 & 3.32 & & 21.31 & 2.83 & & 15.14 & 1.79 & & 31.52 & 3.26 & \\
\hline \multicolumn{17}{|l|}{ HINVESTC } \\
\hline \multirow[t]{3}{*}{ AT } & bajo & 18.29 & 2.82 & & 15.77 & 2.75 & & 20.69 & 2.74 & & 14.6 & 3.23 & & 29.19 & 6.28 & \\
\hline & medio & 18.19 & 3.85 & 0.30 & 14.14 & 3.51 & 0.04 & 19.5 & 5.46 & 0.07 & 14.81 & 2.89 & 0.55 & 30.03 & 6.06 & 0.19 \\
\hline & alto & 19.27 & 2.27 & & 15.83 & 3.49 & & 21.67 & 2.81 & & 15.33 & 2.09 & & 31.6 & 3.67 & \\
\hline
\end{tabular}

Anovap $<0.05$ 
De igual manera se encontró asociación entre el nivel de habilidades investigativas de planificación y ejecución con las subescalas ensayo $(p<0,001)$, elaboración $(p<0,001)$, organización $(p<0,001)$, tiempo y ambiente $(p<0,001)$, regulación del esfuerzo $(p<0,001)$, aprendizaje en paralelo $(p<0,001)$, búsqueda de ayuda $(p<0,001)$, la subescala pensamiento crítico fue significativa en casi todaslas etapas de investigación $(p<0,001)$, ANOVA $(p<0.05)$. (Tabla 3)
Al subcategorizar los datos de ambos grupos a valores altos se encontraron que a mayores valores del CEAM final corresponde a la categoría de habilidades investigativas altas. (Imagen No 5) y al asociar la motivación y estrategias de aprendizaje altas con las habilidades investigativas altas se encontró un RP: $2.82 \mathrm{IC}=(1.55-5.14)$ que indica que una persona con motivación y estrategias de aprendizaje altas tienen casi 3 veces más probabilidades de tener habilidades investigativas altas (Tabla 4).

Tabla 3. Asociación del uso de habilidades investigativas y las subescalas de estrategias de aprendizaje

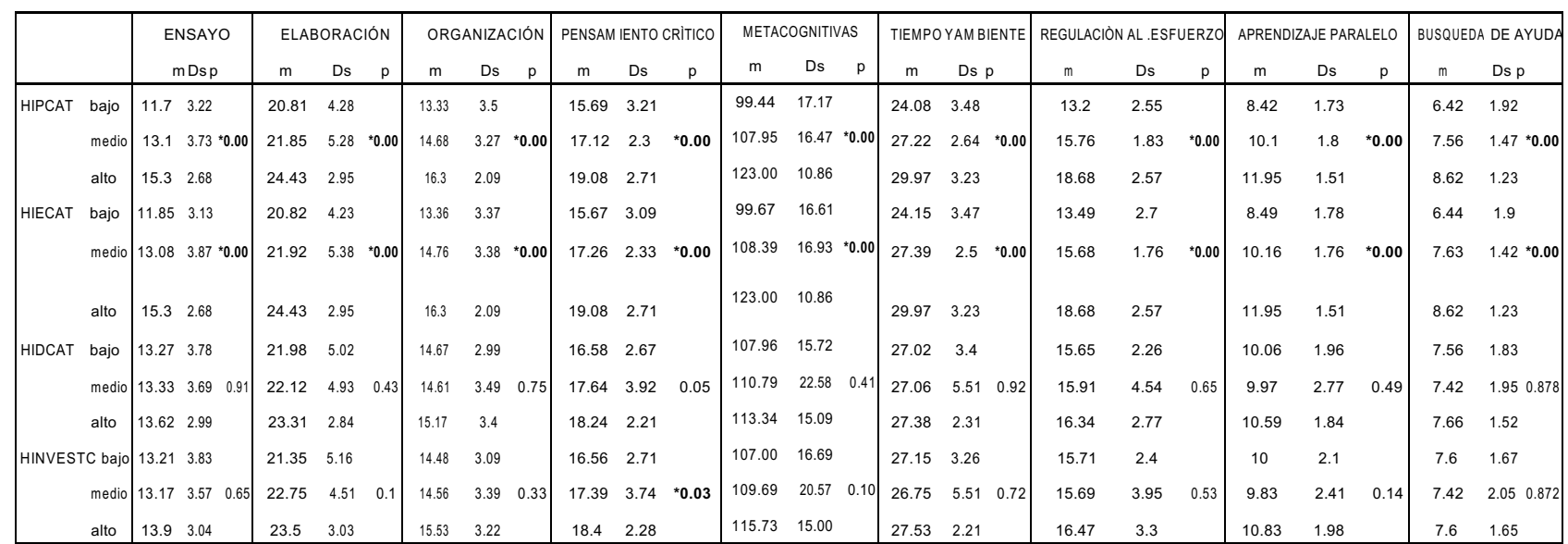

Anovap $<0.05$

HINVEST Habilidades investigativas finales

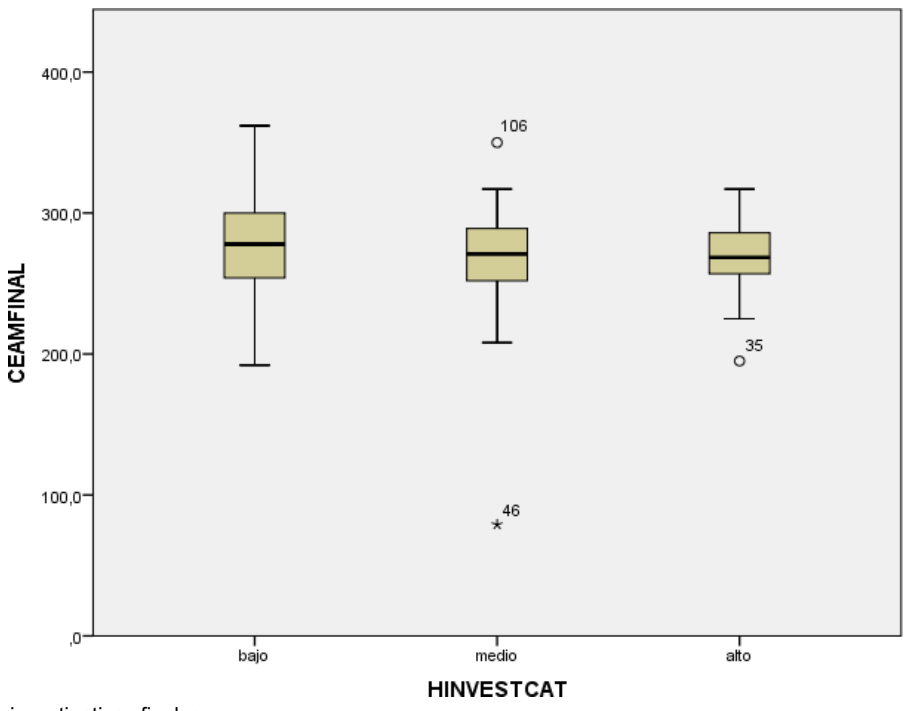

HINVEST Habilidades investigativas finales

Figura 11. Distribución del uso de estrategias de aprendizaje y motivación altas de acuerdo al nivel de habilidades investigativas en estudiantes de posgrado en 
Tabla 4. Razón de prevalencia entre el nivel de motivación y estrategias de aprendizaje altas y las habilidades investigativas altas de los estudiantes de posgrado en odontología de la FO-USMP

\begin{tabular}{|c|c|c|}
\hline & $\mathrm{RP}$ & IC95\% \\
\hline MotEA (alto) vs. Hip (alto) & 2.82 & $1 . \overline{55-5.14}$ \\
\hline $\begin{array}{l}{ }^{*} \text { MotEA Motivación } \\
\text { estrategias de aprendizaje } \\
{ }^{*} \text { Hip Habilidades } \\
\text { investigativas }\end{array}$ & & \\
\hline
\end{tabular}

\section{DISCUSIÓN}

En cuanto a las características educacionales de los estudiantes de posgrado, la mayoría refiere haber realizado trabajos de investigación previos siendo más frecuentes los posters y mesas clínicas con un $45.62 \%$, valores similares a los de Hollman, et al. (9) que menciona que el $63 \%$ de los encuestados había realizado investigaciones antes de matricularse, el $34 \%$ había participado en investigaciónes durante el programa de estudios y con Romesh, et al. que muestra que el $54 \%$ de los estudiantes entrevistados habían presentado sus proyectos de investigación en un foro fuera de Harvard. ${ }^{(8)}$

El porcentaje encontrado en este estudio en referencia a publicaciones científicas realizadas es de $13.15 \%$ a diferencia de lo reportado por Romesh, et al. ${ }^{(8)}$ en USA donde $36 \%$ de los estudiantes tiene su investigación publicada en revistas indexadas.

Solo 22 estudiantes (19.30\%) son asociados a una sociedad científica y el $28.07 \%$ refieren haber llevado adicionalmente cursos de metodología y métodos de estudio, pudiendo ser estos puntos indicadores en el desarrollo e involucramiento en actividades de investigación científica. Más de la mitad de estudiantes maneja el idioma inglés como segunda lengua seguido del portugués y francés. El interés por realizar investigaciones es bastante alto (73.68\%) coincidiendo con Carrillo, et al. (22) donde también un porcentaje similar $(71.2 \%)$ de estudiantes de medicina manifiesta tener intención de dedicarse al campo de la investigación.

Al asociar habilidades investigativas ycaracterísticas sociodemográficas no se encontró significancia $(p<0.05)$, lo que podría indicar que el desarrollo de habilidades investigativas es independiente de las características personales y pueden haber otros puntos que motiven el desarrollo e interés por la investigación científica, como los que menciona Monireh ${ }^{(23)}$ donde los factores externos: ambientales, financiamiento e infraestructura también son importantes; o factores internos, como resalta Imafuku, et al.(24) al realizar una investigación cualitativa en la que los estudiantes dan mayor atención a los procesos de investigación, incluyendo la autonomía del investigador, la colaboración y los procesos de construcción del conocimiento. En cuanto a las características educacionales de los estudiantes se encontró asociación con el ciclo de estudio, trabajos de investigación realizados, cursos previos y participación en una asociación científica, lo que coincide en parte con Harrison y cols. ${ }^{(10)}$ que concluye que si existe diferencia en las habilidades investigativas según el nivel de preparación académica y Lockett, et al. ${ }^{(25)}$ que menciona que los alumnos de años superiores expresan un estilo de aprendizaje más reflexivo y crítico.

Al evaluar el nivel de habilidades investigativas en estudiantes del posgrado en odontología se encontró que la gran cantidad de respuestas favorables señala que poseen un nivel entre medio y alto $(57.88 \%)$; mejores resultados que los querefiere Carrillo-Larco, et al. ${ }^{(22)}$ en Perú donde las habilidades investigativas valoradas por los estudiantes de medicina son limitadas y con el estudio de Rosales, et al. ${ }^{(26)}$ en la habana Cuba que concluyen que más de la mitad de los encuestados valoraron entre regular e insuficiente la preparación recibida.

En cuanto a las etapas de investigación se encontró mejores habilidades investigativas en la etapa de planificación, seguida de la etapa de ejecución y difusión.

Los ítems mejor valorados fueron la formulación del problema de investigación, redacción de objetivos, uso de bases de datos especializadas y manejo de programas de computador, siendo los menos 
valorados: conocimiento de organismos que financian investigaciones, preparar propuestas de investigación para conseguir fondos, conocer el manejo de las guías Strobe, consort, prisma ,como publicar investigaciones como miembro de un equipo de investigación, lo que difiere de Carrillo-Larco, et al. (22) que menciona que las habilidades mejor percibidas por los estudiantes fueron la redacción de un artículo, el diseño de un estudio, y la recolección de datos, mientras que, las habilidades analíticas (cálculo del tamaño de muestra y análisis estadístico) fueron las menos prevalentes lo que coincide con Holman $^{(9)}$ en que las áreas de mejora en los currículos dentales debe ser una formación más rigurosa en metodología de la investigación, revisión crítica de literatura científica y bioestadística y a lo que agrega Keib, et al. ${ }^{(27)}$ que menciona que se debe poner énfasis en la aplicación de la práctica basada en la evidencia. Imafuku(24), sin embargo, coincide con nuestro estudio en el cual hay una tendencia en tener dificultades para llevar a cabo proyectos de investigación en grupo, los cuales el asocia a experiencias de aprendizaje previo, valoración hacia la comunicación interpersonal, la comprensión del proceso de investigación, y las relaciones sociales con los demás.

El cuestionario CEAM completo se valoró como alto con una media de 535. 98, la dimensión motivación como alto ( $\mathrm{x}$ : 197.18) seguido de la dimensión estrategias de aprendizaje como alto (x: 170.89). resultados bastante favorables.

Dentro de la dimensión motivación las subescalas mejor valoradas fueron la subescalas eficacia personal (x: 59.65) y el valor de la tarea (x: 40.93), que es la que conduce al profesional a obtener resultados positivos en su trabajo y tiene mucho que ver con la responsabilidad. La motivación interna fue mejor valorada ( $\mathrm{x}$ : 36.71) a diferencia de la motivación externa con un promedio de 30.28 . lo que quiere decir que los estudiantes realizan acciones únicamente por el placer que les produce la acción en sí misma, no por buscar una recompensa; en coherencia con Monireh S. y cols. ${ }^{(23)}$ que resalta el papel mediador de la autoeficacia en relación a los factores de la investigación y la motivación, Stegers, et al. ${ }^{(28)}$ refiere que para aumentar la autoeficacia, las escuelas de medicina deben ayudar a los estudiantes a monitorear su propio progreso y construir su confianza en su capacidad de aprender, posibles estrategias son: enfocarse, la retroalimentación sobre las competencias dominadas en lugar de sobre aquellos que aún no han sido dominados, proporcionar al estudiante tareas auténticas que se ajusten a su habilidad, desarrollo y crear un aprendizaje "seguro".
En la dimensión Estrategias de aprendizaje lasmejor valoradas fueron las Estrategias Metacognitivas que tienen que ver con la autoconciencia de los procesos mentales, la reflexión sobre la forma en que se aprende, la administración y regulación del uso de estrategias de aprendizaje, observar y analizar desde fuera el propio aprendizaje con autonomía, seguida de las Estrategias de Gestión de Recursos. Dentro de las estrategias metacognitivas sobresalen las subescalas de Metacognitivas, Elaboración y Pensamiento Crítico y dentro de la escala de Gestión de Recursos las subescalas mejor valoradas fueron: las de Tiempo y ambiente y Regulación al esfuerzo; siendo el Aprendizaje en paralelo mejor valorado que la búsqueda de ayuda, lo que coincide con el estudio de Villegas, et al. (12) donde la valoración del estudio que hacen los estudiantes, se relacionan directamente con estrategias de organización y regulación metacognitiva.

Asimismo, se encontró asociación en el nivel de habilidades investigativas de planificación y ejecución con la motivación y con las subescalas: motivación interna, motivación externa, valor de la tarea, confianza del aprendizaje y eficacia del aprendizaje $(p<0.001)$ ANOVA $(p<0.05)$. En coherencia con la evidencia reciente que menciona que los estudiantes intrínsecamente motivados tienen estrategias de estudio de nivel profundo y muestran un aprendizaje conceptual mejorado, creatividad, y flexibilidad cognitiva(28-35).

De igual manera se encontró relación entre el nivel de habilidades investigativas de planificación y ejecución con las subescalas ensayo, elaboración, organización, tiempo y ambiente, regulación del esfuerzo, aprendizaje en paralelo, búsqueda de ayuda, siendo la subescalas pensamiento crítico significativa en casi todas las etapas de investigación $(p<0.001)$, ANOVA $(p<0.05)$ lo que llama la atención y guarda concordancia con lo que dice la teoría en la que se define el pensamiento crítico como una paradoja o reto para la educación en odontología, siendo el arte de analizar un proceso de pensamiento con la intención de mejorarlo dentro de una cultura de educación dental que generalmente enfatiza y valora elementos que son "científicos"; se nutre dela Gestalt del pensamiento complejo y se aplica en todo ámbito; en buscar y criticar la literatura científica, la evaluación del paciente, el tratamiento, planificación, autoevaluación, práctica basada en la evidencia, ética, integración de la tecnología en la práctica, síntesis, gestión, etc. Johnsen, et al. (36) menciona que el pensamiento crítico es un proceso que implica reflexión, duda, investigación, curiosidad y desafío; fundamentalmente es la capacidad de hacer preguntas y de descubrir o crear soluciones que sean razonables y defendibles, encontrar nuevas 
alternativas y percibir varios aspectos de un problema lo que se refleja en el reconocimiento de los propios sesgos, la diferenciación entre hecho y opinión y el refuerzo de la autoevaluación, lo cual nos da una nueva orientación de la enseñanza(36-8)

Se encontró que a mayores valores del CEAM final corresponde a la categoría de habilidades investigativas altas y al asociar la motivación y estrategias de aprendizaje altas con las habilidades investigativas altas se encontró un RP: $2.82 \mathrm{IC}=(1.55$ - 5.14) lo cual se interpreta que una persona con motivación y estrategias de aprendizaje altas tienen casi 3 veces más probabilidades de tener habilidades investigativas altas, lo que coincide con lo desarrollado por Vildoso ${ }^{(17)}$ en el que refiere que si encontró relación en el uso de estrategias de aprendizaje y autoeficacia personal con las habilidades investigativas de estudiantes de maestría en educación de la UNMSM y como lo referido por Visser-Wijnveen, et al. (39) que manifiestan que los factores motivación, reflexión, participación y la investigación actual, capturan con precisión la percepción de los estudiantes sobre la integración de la investigación.

Al igual que Knigth, et al. ${ }^{(40)}$ es importante mencionar que el desarrollo de habilidades investigativas es esencial para lograr profesionales de la salud competentes ya que el proceso de investigación se integra con el aprendizaje sobre la salud de la población y los determinantes sociales en salud pública; por lo que debe encontrarse articulado en el currículo de la carrera de Odontología.

Las principales limitaciones del estudio son implícitas de los diseños observacionales, como el sesgo de información, el cual se minimizó validando los instrumentos y al ser los cuestionarios totalmente anónimos favoreció la veracidad de las respuestas sin ningún temor por parte de los encuestados. Los resultados cuentan con una adecuada validez interna, se puede generalizar a la población de estudio más no a todas las universidades del Perú, por lo que sería necesario realizar una encuesta censal a todas las escuelas de odontología del Perú así como lo realizaron Hollamn, et al. en USA(9). Se recomienda realizar más estudios en los siguientes niveles de evidencia, aplicando diseños mixtos que puedan evaluar la complejidad de todas las variables a nivel inter y multidisciplinario que permitatriangular la información percibida no solo por los estudiantes, sino también por los docentes y autoridades universitarias; diseños experimentales que permitan valorar de manera concreta los productos científicos por parte de docentes yestudiantes así como todo el proceso de enseñanza-aprendizaje implementando métodos activos de enseñanza en investigación científica.

Con el resultado del estudio se concluye que hay interés por parte de los estudiantes del posgrado a involucrase a actividades de investigación científica, siendo su nivel de medio a alto. Por lo que es importante la motivación y uso de estrategias de aprendizaje en la enseñanza de la curricula dental del posgrado, articulando la odontología basada en evidencia y el pensamiento crítico como habilidades fundamentales en el desarrollo de su profesión, cumpliendo así con los estándares internacionales recomendados por la ADA, NIDCR, ADEA, AADR, IADR y NSRG. (Asociación Dental Americana, en asociación con the National Institute of Dental \& Craniofacial Research, The American Dental Education Association, The American Association for Dental Research, International Association for Dental Research and the National Student ResearchGroup)

Agradecimientos: A los estudiantes de posgrado de la FO-USMP por su participación en el estudio y al Doctor Roberto León Manco por su asesoría estadística en el presente trabajo.

Participacion de autoria: DAC, AQA diseñaron el estudio. DAC, JGC y RMV participaron en la recolección de datos y analisis estadístico. HMO realizo la revisión critica del articulo. Todos los autores aprobaron la redacción final del articulo. .

Conflicto de interés. Los autores declaran no tener conflictos de interés

Fuente de financiamiento: autofinanciado por la autora principal del estudio.

\section{REFERENCIAS BIBLIOGRÁFICAS}

1. Alrahla A. The impact of motivational factors on research productivity of dental faculty members: A qualitative Study. Journal of Taibah University Medical Sciences. 2016; 11(5): 448-455.

2. Accreditation standards for dental education programs. American dental association (2013). URL accessed on 10-01-15 at: http:www.ada.org-section-education and carerees-pdf-predoc-2013.pdf.

3. Emrick J, Gullard A. Integrating research into dental student training: a global necessity. J Dent Res. 2013; 92(12):1053-55.

4. Sistema nacional de Evaluación, Acreditación y Certificación de la Calidad Educativa. Acreditación. Lima: SINEACE; 2016. 
5. SINEACE. Estándares para la Acreditación de la Carrera Profesional Universitaria de Odontología. Lima: Diario Oficial El Peruano; 12 de julio de 2010.

6. Jaik A, Ortega E. El nivel de dominio de las competencias en metodología de la investigación que poseen los alumnos de posgrado. (Tesis doctoral). Instituto Universitario Anglo Español.España.2012.

7. Rojas $M$, Vasquez $C$, Roque M. Las competencias investigativas en la construcción del talento humano dentro de las ciencias médicas. Med ciego.2012; 18(2).

8. Romesh P.N, Min Kyeong L, Da Silva J, Veerasathpurush A. Impact of a Research Requirement in a Dental School Curriculum. J Den Educ.2014; 78:1364-1371.

9. Holman SD, Wietecha M, Gullard A, Petersen J. Research experiences impact U.S. dental students positive attitudes toward research and science. J Dent Educ. 2014; 78(3):334-348.

10. Harrison L, Hernández A, Cianelli R, Rivera M, Urrutia $M$. Competencias en investigación para diferentes niveles de formación de enfermeras: una perspectiva latinoamericana. Rev Cienc Enferm. 2005; 12.

11. Canto M, Cabrera A, Franco M. El desarrollo de habilidades investigativas en estudiantes de estomatología, dimensión necesaria para una formación integral.Edumecentro.2014;6:166-72.

12. Villegas G, Sotelo L, Sotelo N, Dominguez S. Relación entre estrategias de aprendizaje y motivación en una muestra de estudiantes universitarios de Lima Metropolitana. Rev Psicol. 2011; 3(3): 31-44.

13. Estrada-Valenzuela C, Prado-Mendoza J, De la FuenteCabrera L, Navarro-Villalobos M, Elizalde-Corona A, Aguirre-Sarabia N. Eficacia de estrategias de aprendizaje utilizadas por alumnos de la Facultad de Odontología de la Universidad Autónoma de Coahuila, Unidad Torreón. Rev Tamé.2014; 3(8):264-270.

14. Salim R, Lotti de Santos M. Evaluación de enfoques, motivaciones y estrategias de aprendizaje en estudiantes del primer año universitario de odontología. Cuadernos de educación. 2011; 9.

15. Bahamón M, Vianchá Pinzón M. Estilos y estrategias de aprendizaje: una revisión empírica y conceptual de los últimos diez años. Rev Pens Psicol. 2012; 10 (1):129144.

16. Calderón L, Chiecher A. Uso de estrategias de aprendizaje en estudiantes universitarios y estudiantes de maestría. (Tesis doctoral). Universidad nacional de rio cuarto. Argentina.2011.

17. Vildoso J. Estrategias de Aprendizaje y Autoeficacia en el Desarrollo de Habilidades investigativas de los Maestristas de la Facultad de Educación de la UNMSM. (tesis para optar el grado de doctora en educación).Universidad Nacional Mayor de San Marcos. Lima-Perú.2010.

18. Freiberg Hoffmann A, Ledesma R, FernándezLiporace R. Estilos y estrategias de aprendizaje en estudiantes universitarios de Buenos Aires. Rev de Psicolog.2017; 35 (2), 2017.

19. McAndrew M, Morrow C, Atiyeh L, Pierre G. Dental Student Study Strategies: Are Self-Testing and Scheduling Related to Academic Performance?. J Dent Educ. 2016; 80(5):542-52.

20. Alfaro D, Evaristo T, Ayala G, Palomino C, León R, Quitzgaard A. Motivación, estrategias de aprendizaje y su relación con el nivel de habilidades investigativas en estudiantes de posgrado. Un estudio piloto. Odontol San Mar. 2017; 20(1):7-12.

21. Montoya O. Application of the factorial analysis to the investigation of markets. Case of Study. Scientia et Technica.2007;(35):281-86.

22. Carrillo-Larco R, Carnero A. Autoevaluación de habilidades investigativas e intención de dedicarse a la investigación en estudiantes de primer año de medicina de una universidad privada en Lima, Perú. Rev Med Hered. 2013; 24:17-25.

23. Monireh S, Hossein K, Mohammad Reza A. The Role of Social- Cognitive References in Academic Situations on Students' Research Self-Efficacy and Research Motivation: Testing a Causal Model. Am J Edu Res. 2013; 1(3): 79-85

24. Imafuku R, Saiki T, Kawakami C, Suzuki Y. How do students' perceptions of research and approaches to learning change in undergraduate research?. Int J Med Educ.2015;12(6):47-55. doi: 10.5116/ijme.5523.2b9e.

25. Lockett M, Ojeda C, Aguirre G, Alicia G, Guarnieri M, Carolina, Vallejos M. Identificación de estrategias y estilos de aprendizaje de los estudiantes de Odontología. FOUNNE. Disponible en: http://odn.unne.edu.ar/at12008-7.pdf.

26. Rosales S, Ruano M, Padrón E, Valverde O, Sanz T. Diagnóstico de la formación investigativa en la carrera de estomatología de la facultad Raúl Gonzales Sánchez. Rev Cubana de Estomatol. 2013; 49(2):226238.

27. Keib C, Cailor S, Kiersma M. Chen A. Changes in nursing students' perceptions of research and evidencebased practice after completing a research course. Nurse Education Today. 2017; 54:37-43.

28. Stegers-Jager $M$, Cohen-Schotanus $A$, Themmen. Motivation, learning strategies, participation and medical school performance. Medical Education 2012; 46: 678-688. doi:10.1111/j.1365-2923.2012.04284.x.

29. Orsini C, Evans P, Binnie V, Ledezma P, Fuentes F. Encouraging intrinsic motivation in the clinical setting: teachers' perspectives from the self-determination theory. Eur J Dent Educ. 20 (2016) 102-111.

30. Ryan RM, Deci EL. Self-determination theory and the facilitation of intrinsic motivation, social development, and well-being. Am Psychol. 2000; 55(1):68.

31. Baker SR. Intrinsic, extrinsic, and amotivational orientations: their role in university adjustment, stress, 
well-being, and subsequent performance.Curr Psychol. 2004; 23(3):189-202.

32. Dos Santos BF, Nicolau B, Muller K, et al. Brazilian dental students' intentions and motivations towards their professional career. J Dent Educ. 2013; 77 (3):337- 44.

33. Aggarwal A, Mehta S, Gupta D, et al. Dental students' motivations and perceptions of a dental professional career in India. J Dent Educ. 2012; 76(12):1532-9.

34. Kusurkar R, Ten Cate T, Vos C, et al. How motivation affects academic performance: a structural equation modelling analysis. Adv Health Sci Educ Theory Pract. 2013; 18:57-69.

35. Vincent Donche, Sven De Maeyer, Liesje Coertjens, Tine Van Daal, Peter Van Petegem. Differential use of learning strategies in first-year higher education: The impact of personality, academic motivation, and teaching strategies. Br J Educ Psychol .2013; 83, 238251.

36. Johnsen D, Mitchell J, Finkelstein M, Marsha A, Cunningham-Ford. Guiding Dental Student Learning and Assessing Performance in Critical Thinking with Analysis of Emerging Strategies. J Dent Educ. 2012; 76(12):1548-1558.
37. Marshall T, Marchini L, Cowen H, Hartshorn, Julie J, Holloway A, Straub-Morarend CH, Gratton D, Solow D, Colangelo N, Johnsen D. Critical Thinking Theory to Practice: Using the Expert's Thought Process as Guide for Learning and Assessment. J Dent Educ. 2017 81:978-985.

38. Hong B, Plugge E.Critical appraisal skills teaching in UK dental schools. Br Dent J. 2017;222:209213.doi:10.1038/sj.bdj.2017.126.

39. Visser-Wijnveen $G$, van der Rijst R, van Driel J. A questionnaire to capture students' perceptions of research integration in their courses. High Educ.(2016) 71: 473. doi: 10.1007/s10734-015-9918-2.

40. Knight SE, Van Wyk JM, Mahomed S. Teaching research: a programme to develop research capacity in undergraduate medical students at the University of KwaZulu-Natal, South Africa. BMC Med Educ. 2016 Feb 16;16:61. 